\title{
The Downside of Globalization
}

\section{Dear Reader,}

Covid-19 will on the one hand have a direct impact on the global economy, but on the other can also be seen as a warning shot across our bows. It has become clear that our systems are not designed to function independently for longer periods. To make matters worse, our supply chains are too one-sided and much too long. If there is one lesson we can learn from the current situation, it is that we must ensure that we do not rely on only one source of supply. This means that when one production site becomes unavailable, there are others we can use. Admittedly this is likely to lead to the products becoming more expensive, but production shutdowns cost a lot more.

We urgently need to apply the same principle as we set out on new paths, including decentralized electricity generation, the use of renewable energy and the construction of plants to manufacture alternative fuels. There seems to be a consensus that electricity should be generated by solar farms in sweet spots in North Africa and Australia. The energy can then be stored there in the form of gaseous or liquid fuels that can be transported relatively easily. It would be a mistake to centralize the generation facilities or to use specific processes exclusively in certain regions. The result of this would be new dependencies of the kind that we are already familiar with in the case of the supply of crude oil from the Middle East. The opportunities and benefits will be much greater if several different countries make use of all the possibilities for electricity generation from electrolysis through to fuel production.
Volkswagen's decision to stop the production of vehicles with CNG powertrains in the medium term, despite the fact that the company announced a product campaign as recently as 2019 , presents a problem for the imminent launch of synthetic fuels onto the market and the move toward technology neutrality. Admittedly, sales of CNG cars have been sluggish, but the policymakers have missed out on a simple and effective opportunity to prevent large quantities of $\mathrm{CO}_{2}$ from being emitted.

Let's hope that this does not come back to bite us in a few years' time, just as our overlong supply chains are doing at the moment.

I hope you enjoy reading this issue of MTZ.

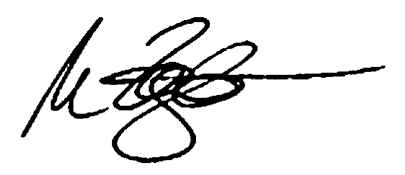

Marc Ziegler

Deputy Editor in Chief

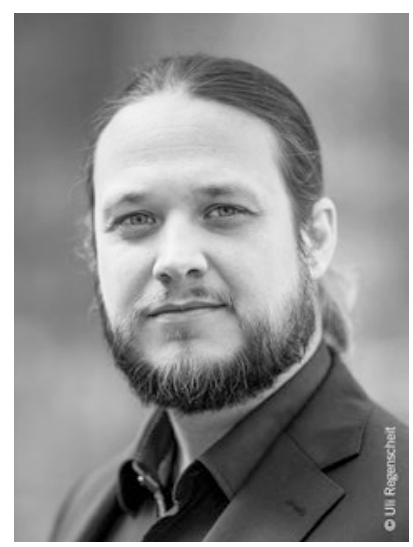

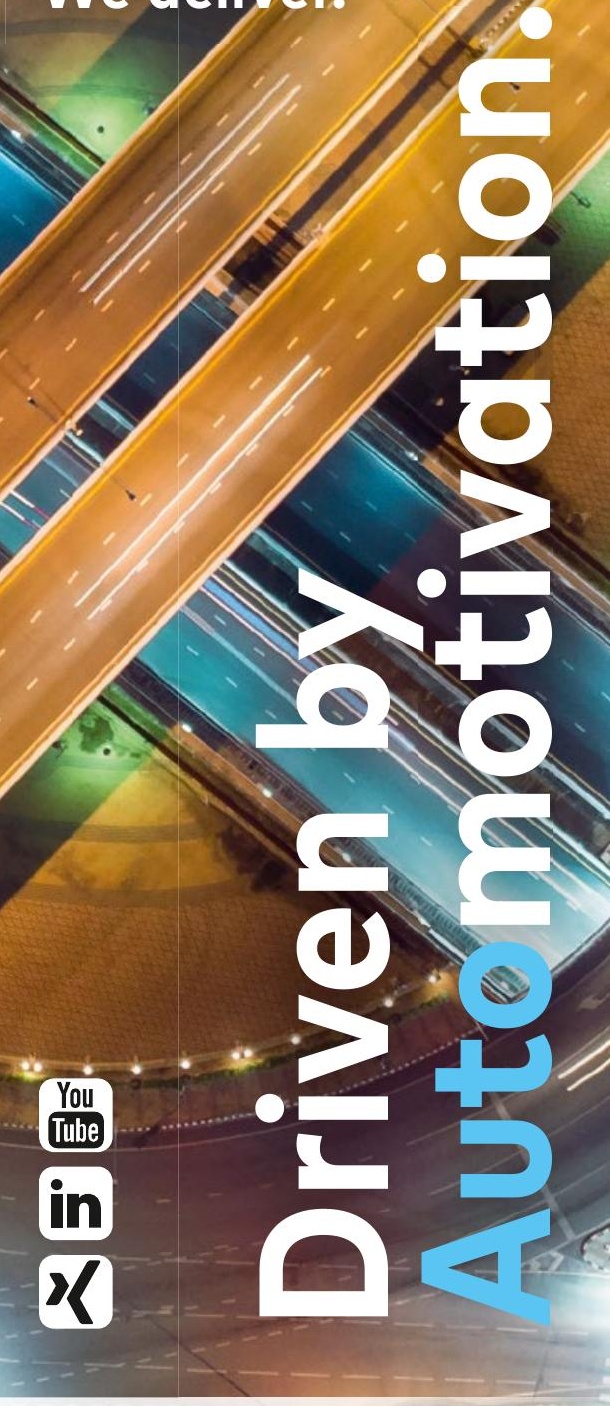

\section{Family owned. $100 \%$ committed.}

